\title{
Based on Kalman Filter Fuzzy PID Control Yuntai
}

\author{
Youqiang Lai 1, a \\ 1 Xijing University, \\ Shaanxi Xi'an ,China \\ a E-mail: 2571449451 @qq.com \\ Xiaoping Yang 3, c \\ 3Shaanxi step industrial co., LTD, \\ Shaanxi Xi'an ,China \\ cE-mail: 41564103@qq.com
}

\begin{abstract}
This paper first introduces the algorithm of Kalman wave. Because random disturbance noise is uncertain, unpredictable, and the mean and variance of noise, for this kind of interference, taking maximum a posteriori (MAP) noise estimator is Sage - Husa to estimate the disturbance of the system noise and measurement noise mean and variance, to realize the Kalman filter for uncertain signal suppression. Then the adaptive Kalman filtering algorithm is combined with fuzzy PID control algorithm, and it is proposed based on adaptive Kalman filter model PID control algorithm, which has both fuzzy PID adaptive control system parameters, fast tracking performance, but also it has the adaptive Kalman filtering jamming signal inhibitory effect of uncertainty. At last, through simulation experiment, results show that the fuzzy PID control based on adaptive Kalman filtering algorithm with fast response speed, high precision, good robustness, and can effectively overcome all kinds of jamming noise existing in the system.
\end{abstract}

Keywords-Kalman;Sage-Husa;PID control algorithm; simulation

\section{INTRODUCTION}

Classic PID of simple structure, high reliability, good stability, for to establish accurate mathematical model of system is particularly useful.But its adaptability is bad, especially for nonlinear system and time-varying system, a classic PID is difficult to achieve ideal control effect. Fuzzy PID controller can be according to the error and error change rate to adjust PID parameters in real time, little overshoot, fast response speed, good performance. But for interference noise, and their inhibitory effect is not obvious, and can't effectively isolate carrier of all kinds of noise, so you need to introduce the effective filtering algorithm. The limitations of the classical Kalman filtering is the requirement for precise mathematical model and noise statistical information (the mean and variance of noise).But in the process of unmanned aerial vehicle (uav) yuntai control, noise factor is complicated, the mean and variance is unknown, for dealing with such filtering problem, raises the adaptive Kalman filtering, using adaptive Kalman filter to suppress control interference existing in the system noise and measurement noise.

\author{
Jingfeng He2,b \\ 2Xijing University, \\ Shaanxi Xi'an ,China \\ bE-mail:573825625@qq.com \\ Min Ji 4, d \\ 4 Xijing University, \\ Shaanxi Xi'an ,China \\ cE-mail: 995010771@qq.com
}

\section{THE KALMAN FILTER}

Unmanned aerial vehicle (uav) three axis stabilized yuntai is influenced by various environmental factors, in order to improve the control accuracy, this paper use the adaptive kalman filter to suppress interference noise and measurement noise control.

System of the discrete model of state equation and measurement equation are:

$$
\begin{gathered}
X(k+1)=\Phi(k+1, k) X(k)+B(k) U(k)+W(k) \\
y_{v}(k+1)=H X(k+1)+V(k) \\
X(k) \_ \text {the state matrix; } \\
y_{v}(k) \_ \text {the observation matrix; } \\
U(k) \_ \text {the control output; } \\
\Phi \_ \text {the state transition matrix; } \\
B \_ \text {the input matrix; } \\
H_{W} \text { the measure transfer matrix; } \\
W(k) \_ \text {control interference noise; } \\
V(k) \_ \text {the measurement noise. }
\end{gathered}
$$

The noise statistics are:

$$
\begin{aligned}
& E\{W(k)\}=q, e\left\{W(k) W^{T}(j)\right\}=Q(k) \delta_{k j} \\
& E\{V(k)\}=r, E\left\{V(k) V^{T}(j)\right\}=R(k) \delta_{k j} \\
& \delta_{k j} \text { is the crow-nick function. }
\end{aligned}
$$

$$
\delta_{k j}=\left\{\begin{array}{l}
1, k=j \\
0, k \neq j
\end{array}\right.
$$

Usually this kind of random disturbance is uncertain, unpredictable, and the mean and variance of noise. For this kind of interference, this paper take the maximum a posteriori (MAP) Sage - Husa noise estimator to estimate the control means, variances of interference noise, measurement noise mean and variance.

Algorithm steps are as follows:

$$
\stackrel{\Lambda}{X}(k+1 \mid k)=\Phi(k+1 \mid k) \stackrel{\Lambda}{X}(k \mid k)+B(k) U(k)+q(k)
$$


$\stackrel{\Lambda}{X}(k+1 \mid k)$ covariance

$P(k+1 \mid k)=\Phi(k+1 \mid k) P(k \mid k) \Phi_{T}(k+1 \mid k)+Q(k)$

Kalman gain

$M(k+1)=P(k+1 \mid k) H_{T}(k)\left[H(k) P(k+1 \mid k) H_{T}(k)+R(k+1)\right]^{-1}$

The information in the Kalman filter items:

$\varepsilon(k+1 \mid k)=y_{v}(k+1)-H(k) \stackrel{\Lambda}{X}(k+1 \mid k)-r(k)$

The optimal estimate of $(\mathrm{k}+1)$ time:

$\stackrel{\Lambda}{X}(k+1 \mid k+1)=\stackrel{\Lambda}{X}(k+1 \mid k)+M(k+1) \varepsilon(k+1 \mid k)$

The corresponding covariance of $\stackrel{\wedge}{X}(k+1 \mid k+1)$ :

$P(k+1 \mid k+1)=[I-M(k+1) H(k)] P(k+1 \mid k)$

$q(k)=\left(1-d_{k}\right) q(k-1)+d_{k}[X(k \mid k)-\Phi(k \mid k-1) X(k-1 \mid k-1)-$

Below with Sage - Husa time-varying noise estimator to estimate $q(k), Q(k), r(k), R(k)$

$$
\begin{aligned}
& Q(k)=\left(1-d_{k}\right) Q(k-1)+d_{k}\left[M(k) \varepsilon(k \mid k-1) \varepsilon^{T}(k \mid k-1) M^{T}(k)\right. \\
& \left.+P(k \mid k)-\Phi(k \mid k-1) P(k-1 \mid k-1) \Phi^{T}(K \mid K-1)\right] \\
& Q(k)=\left(1-d_{k}\right) Q(k-1)+d_{k}\left[M(k) \varepsilon(k \mid k-1) \varepsilon T(k \mid k-1) M_{T}(k)\right. \\
& +P(k \mid k)-\Phi(k \mid k-1) P(k-1 \mid k-1) \Phi_{T}(k \mid k-1) \\
& r(k)=\left(1-d_{k}\right) r(k-1)+d_{k}\left[y_{v}(k)-H(k) X(k \mid k-1)\right] \\
& R(k)=\left(1-d_{k}\right) R(k)+d_{k}\left[\varepsilon(k \mid k-1) \varepsilon^{T}(k \mid k-1)-H(k) P(k \mid k-1) H^{T}(K)\right] \\
& 0<\mathrm{b}<1 \text { is forgetting factor, to the test to determine, }
\end{aligned}
$$
usually preferable to 0.95 or 0.99 .

\section{THE FUZZY PID CONTROL BASED ON KALMAN FILTER}

Adaptive Kalman filtering and the combination of fuzzy PID controller, make full use of robust fuzzy control and PID control error is small, the advantages of the fuzzy reasoning method to realize online self-tuning of PID parameters used. At the same time the adaptive Kalman filter to filter the signal, it reduces all kinds of jamming noise yuntai system, and makes the whole system that has good adaptability.

Fuzzy PID control based on adaptive Kalman filtering structure is as follows:

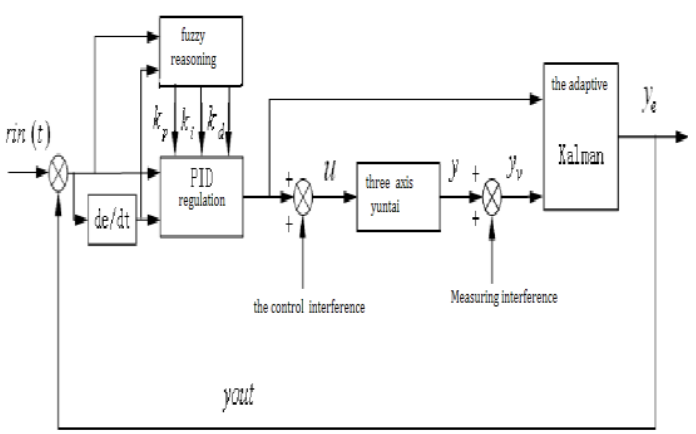

Figure 1. fuzzy PID control algorithm based on adaptive Kalman filtering structure

\section{YUNTAI FUZZY PID CONTROL SIMULATION BASED ON KALMAN FILTER}

Simulation conditions and parameters as before, the fuzzy PID control algorithm based on Kalman filtering simulation experiment, and the simulation results with the classic PID control algorithm and fuzzy PID control algorithm were compared.The simulation results are shown in Fig .2. We can see out from the table that the classic PID control algorithm is some at the beginning of the step response overshoot, and it has reached a stable state during 0.025 s. Fuzzy PID control algorithm and fuzzy PID control algorithm are based on Kalman filter response speed, and control relatively stable, no overshoot, basic to achieve stable during $0.02 \mathrm{~s}$, the response speed of the fuzzy PID based on Kalman filter response slightly faster than the fuzzy PID, the advantage is not obvious.

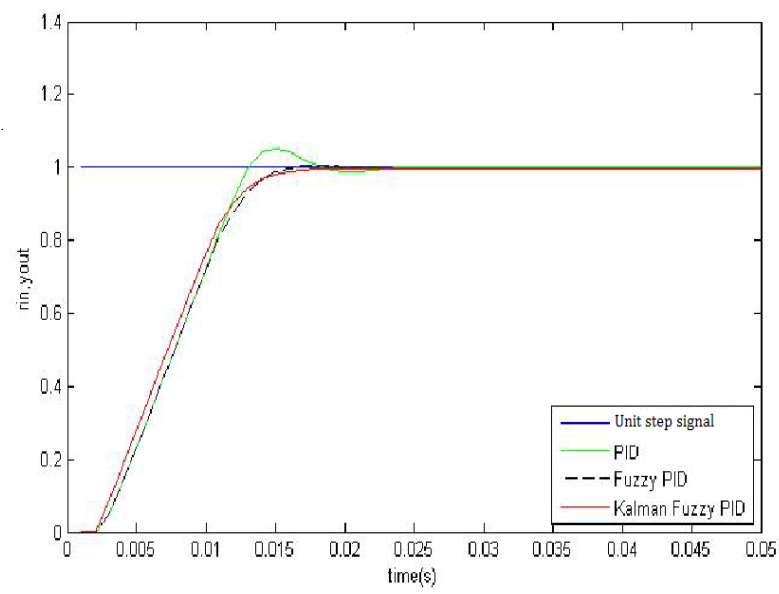

Figure 2. without noise step signal simulation of three kinds of control algorithm

As shown in Fig .2. it is adopted in the system when the white noise amplitude of 0.1 . The simulation results show that the classic PID and fuzzy PID although response quickly, but in a small shock after the stability, unable to effectively overcome the interference noise and measurement noise control, and it can't achieve ideal control effect. The fuzzy PID control algorithm based on Kalman filtering is still fast response speed, quickly reached a stable state, and there is no steady-state error, the whole system is not affected by noise. It improves the system stability, effectively reduces the system error, and achieved good control effect.

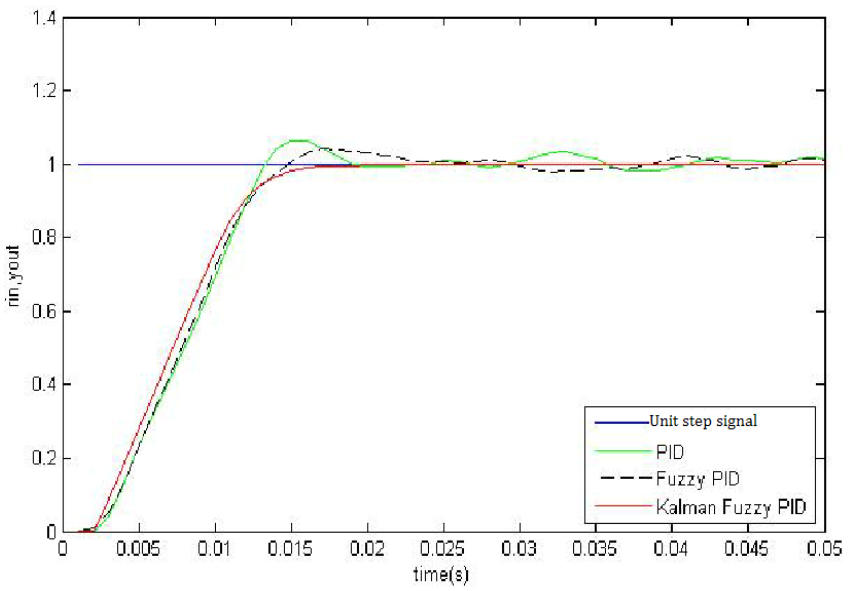

Figure 3. white noise amplitude of 0.1 cases step signal simulation of three kinds of control algorithm 
As shown in Fig .4 and 5 are the three kinds of control algorithm simulation results of sine wave, which in Fig .4.add the white noise amplitude of 0.1, Fig .5. is to join the white noise amplitude of 0.1.Simulation results show that when the noise amplitude is not big, the classic PID and fuzzy PID control error is not very obvious, the basic can achieve stability control and tracking, but when the noise amplitude is larger, the control accuracy will fell sharply with the increase of noise amplitude, affected the normal work of the yuntai. Fuzzy PID control algorithm based on Kalman filtering control effect is not with the increase of noise down, and it still can maintain higher control precision, and can satisfy the requirements of the system noise suppression interference.
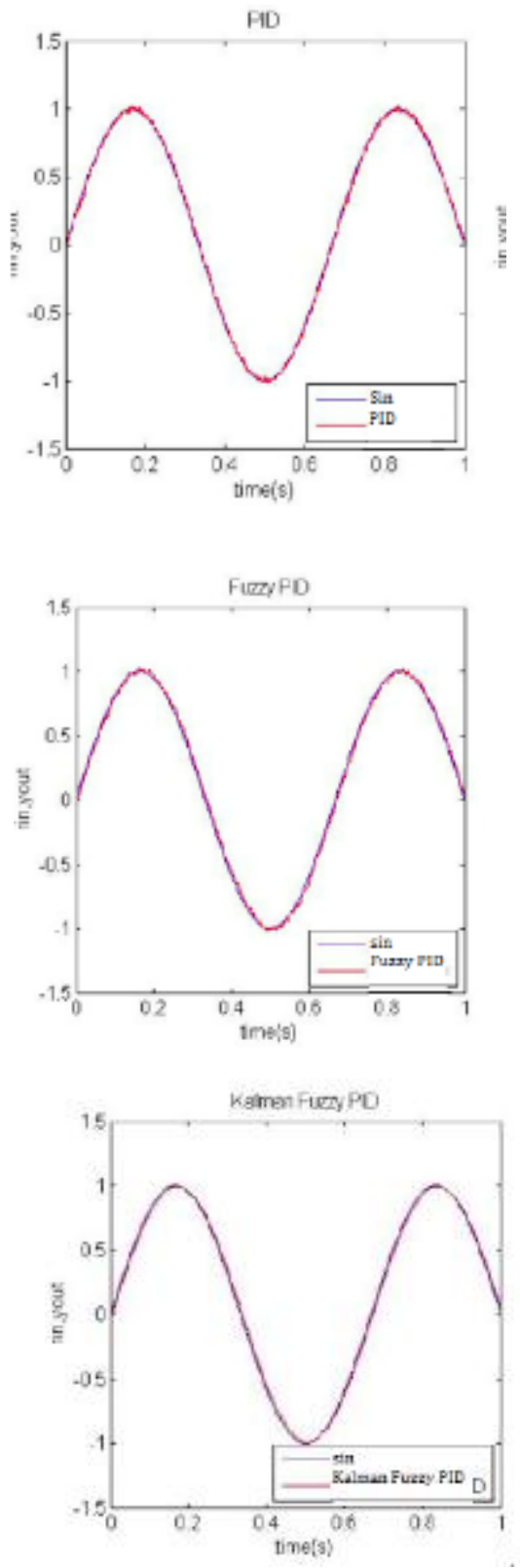

Figure 4. white noise amplitude of 0.01 cases sine wave simulation of three kinds of control algorithm
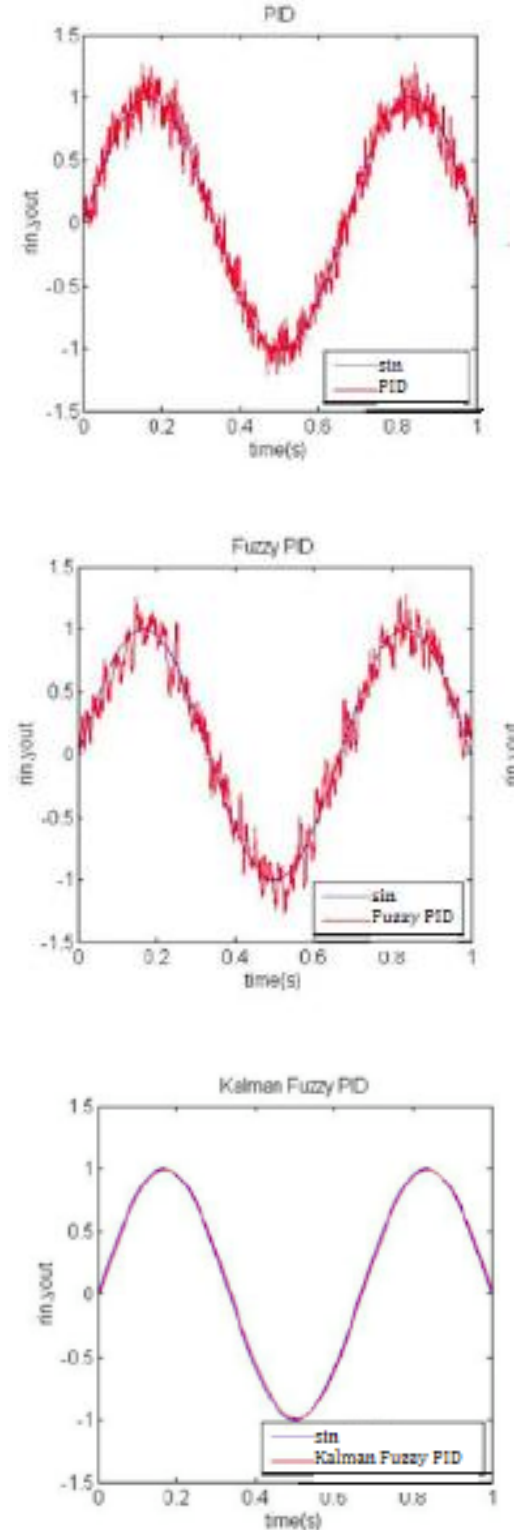

Figure 5. the white noise amplitude of 0.1 cases of three kinds of sine wave simulation results of the algorithm

\section{CONCLUSIONS}

The above simulation analysis shows that fuzzy PID control algorithm based on adaptive Kalman filter can effectively overcome all kinds of nonlinear interference factors existing in the system, with less overshoot, short setting time, high control precision, good robustness etc, and it has good application value.

\section{REFERENCES}

[1] Rick Walter, Harrison Danny, Jed Donaldson, etc. Stabilized Inertial Measurement System(SIMS)[C]// Proceedings of SPIE Laser Weapons Technology III,2002:57-68.

[2] Valerie Paul, Benot Ricard, Andre Zaccarin. Step-stare technique for airborne high-resolution in frared imaging[J].SPIE-The international Society for Optical Engineering,2004,04:128-138.

[3] Slobodan N. Vukosavic. Suppression of Torsional Oscillations in A High-Performance Speed Servo Drive[J].IEEE Trans. on Industry Electronics.1998,45(1):108-117.

[4] Marcelo C A, Douglas E E. Novel Kalman Filtering Method for the Suppression of Gyroscope Noise Effects in Pointing and Tracking Systems[J].Optical engineering.1995,34(10):3016-3030. 
[5] Bo Li, D Hullender, M DeRenzo. Nonlinear Induced Disturbance Rejection in Inertial Stabilization Systems[J].IEEE Trans. on Control System Technology.1998,6(3):421-427

[6] J.A.R. Krishna Moorty, Rajeev Marathe, V.R. Sule'. HœControl Law for Line-of-sight Stabilization for Mobile Land Vehicles[J]. Optical Engineering. 2002,41(11):2935-2944.

[7] Willian J. Bigley, Steven P. Tsao. Optimal Motion Stabilization Control of an Electro-Optical Sight System[C]//Proc of SPIE.1989:116-120.
[8] Ho W K, Hang C C, Cao L S. Tuning of PID controller based on gain and phase margin specification[J].Automatic,1995,31(3):497502.

[9] Stuartr Bennett. The past of PID controller[J].Annual Reviews in Control,2001,25:43-53.

[10] Shi Y H, Eberhart R C. A Modified Particle Swarm Optimization[C]//Proc of 1998 IEEE International Conference on Evolutionary Computation, Anchorage, Alaska,May4-9,1998:6973. 